\title{
Patient recall versus physician documentation in report of smoking cessation counselling performed in the inpatient setting
}

Jessica M Nicholson, Deborah J Hennrikus, Harry A Lando, Maribet C McCarty, John Vessey

\begin{abstract}
Objectives-To determine rates of patient reported and physician documented counselling; to identify predictors of each report; and to identify the impact of each report on smoking cessation attempts after discharge from the hospital.

Design-Stickers on subjects' charts prompted physicians to give brief smoking cessation counselling to patients in the hospital. Patients reported counselling received and quit attempts in a phone interview conducted 7-18 days after discharge. Rates of counselling and correlations were calculated, and multivariate analysis identified predictors of patient report, physician documentation, and quit attempts.
\end{abstract}

Setting-Four hospitals in the Minneapolis/St Paul metropolitan area. Subjects-682 hospital patients who had smoked more than 100 cigarettes in their lifetime and had smoked in the last three months.

Results-71.0\% of patients reported counselling, and physicians documented counselling in the charts of $46.2 \%$ of patients (correlation $=0.15, \kappa=0.13)$. Patient report was predicted by specific hospital, belief that their hospitalisation was smoking related, diagnosis of a smoking related disease, and physician documentation of counselling. Physician documentation was predicted by female patient, specific hospital, longer hospital stay, and marginally predicted by smoking related disease. Quit attempts were predicted by patient report of counselling, but not physician documentation.

Conclusions-Physicians document counselling in the hospital at a lower rate than patients report it, and the correlation between reports is very low, making an accurate assessment of true rates of counselling difficult. While it is important to increase physician documentation, it is even more important to increase patient recall, as this is the only report that predicts a quit attempt.

(Tobacco Control 2000;9:382-388)

Keywords: smoking cessation counselling; patient recall; physician documentation

For many smokers, a hospital stay presents a "teachable moment". ${ }^{1}$ Smokers are not allowed to smoke in the hospital, which forces many to quit temporarily. Additionally, the illness leading to their hospitalisation can often motivate them to consider quitting permanently. Most importantly, they have access to health professionals who can advise them about their smoking, at a time when they are likely to respond.

Studies show that physicians may not be taking advantage of the opportunity to intervene with hospitalised smokers. In an exit questionnaire with hospitalised smokers, $66 \%$ of patients on a cardiovascular disease unit and $17 \%$ on a general medical unit reported receiving smoking cessation advice from their physician. ${ }^{1}$ Similarly, in a survey mailed to smokers after discharge, only $68 \%$ reported receiving any advice. ${ }^{2}$ Even with prompts to remind physicians to give advice in the hospital, the rate of patient reported advice is low, ranging from $49-55 \% .^{34}$

Evidence from the outpatient setting suggests that prompts can be an effective means of increasing smoking cessation counselling. For example, when smoking status was documented as a vital sign, patients reported that discussion of smoking increased from $47 \%$ to $86 \%$ and that physician advice to quit increased from $50 \%$ to $80 \%{ }^{5}$; these findings are consistent with those of similar studies. ${ }^{6}$ Given this success, chart reminders have been proposed for use in the hospital environment as well.

When physicians counsel patients about smoking cessation, they may favour particular subgroups. For example, reports of advice increased from $55 \%$ among smokers overall to $77 \%$ and $72 \%$ among patients with pulmonary disease and cardiovascular disease, respectively. ${ }^{3}$ Other authors have similarly found that patients with smoking related diseases are more likely to report advice. ${ }^{7-9}$ Reports of advice were also greater with patient perception of poor health, ${ }^{10}{ }^{11}$ increased readiness to quit, ${ }^{11}$ and female sex. ${ }^{812} 13$ However, while these characteristics may make it more likely that patients will receive advice from physicians, they may also be characteristics that increase the likelihood that a patient will report advice.

In the outpatient setting, several studies have found that patients over report and/or physicians under document smoking cessation counselling. When compared to audiotape, smokers correctly reported physician counsel- 
ling at rates ranging from $92-98 \%$, with an approximately $20 \%$ false positive rate. ${ }^{14}$ In another study, patients correctly reported advice heard on audiotape at a rate of $81.8 \%$, with a false positive rate of $10.5 \% .{ }^{15}$ Physicians documented only $45.5 \%$ of smoking advice heard on audiotape, although chart notes were found to have a $0 \%$ false positive rate. ${ }^{15}$ These patterns have been found in other studies as well. ${ }^{16-20}$ The issue is further complicated by poor correlation between the two reports, with one study finding that only $44.4 \%$ of smokers who reported advice had a corresponding physician's note in their chart. ${ }^{15}$ When measuring physician counselling behaviours, patient interview and chart audit have been found to agree the least when compared to videotape. ${ }^{21}$

This phenomenon of patient over report and physician under documentation raises issues in any study that attempts to measure smoking cessation counselling given to patients using either report. This is complicated by the fact that the accepted "gold standards", such as audiotaping, are so logistically difficult as to be prohibitive in any large scale study. This report uses data from the Teachable Moment (TEAM) study, a randomised controlled trial of the effectiveness of smoking cessation counselling with hospital inpatients, to explore issues of patient recall and physician documentation of counselling. This study aims to identify: (1) rates of counselling reported by patients and charted by physicians in the hospital and their correlation; (2) independent predictors of each report of counselling; and (3) the impact of physician documented and patient reported counselling on smoking cessation attempts by patients immediately after discharge from the hospital. While several studies have examined this issue in the outpatient setting, the present study is one of the first to look at reports of smoking cessation counselling in the inpatient environment. Furthermore, to our knowledge, this is the first study to identify predictors of physician documentation.

\section{Methods}

SETTING

Four hospitals participated in this study. All are located in the Minneapolis/St Paul metropolitan area. Hospitals $\mathrm{A}$ and $\mathrm{C}$ are both urban hospitals with large minority and transient populations. Hospitals B and D are suburban, and Hospital $\mathrm{B}$ has a lower proportion of minority patients than the other hospitals. Hospital A is publicly owned, while the other hospitals are operated by managed care organisations.

\section{SUBJECTS}

Subjects were patients admitted to the four study hospitals who had smoked more than 100 cigarettes in their lifetime and had smoked in the past three months. This latter criterion was included in order to capture patients who had recently stopped smoking owing to illness or in anticipation of hospitalisation. Patients were excluded if they were not between the ages of 18 and 75 years, were not admitted for at least 24 hours, were obstetric or psychiatric patients, were too ill or impaired to participate, were assigned to a room with another patient in the study, or were not available for follow up for a period of 12 months (no phone, non-English speaking, in detention). Patients were recruited between January 1997 and June 1999.

SMOKING CESSATION PROGRAMME

Consenting patients were randomly assigned to one of three treatment conditions: minimal intervention, brief provider advice (PA), or provider advice plus nurse counselling. All patients were given the TEAM smoking cessation booklet by the research assistant. Only patients in the PA intervention were used for this analysis, as it was the only group in which provider counselling was documented, but no nurse counselling took place that might bias patients' recall of physician counselling specifically. The PA intervention consisted of labelling the patients' charts. The label was placed prominently in the progress notes and read: "Physicians: this patient is a SMOKER who has agreed to participate in the TEAM smoking cessation study. Please give a brief ( 60 second) message that includes the following: advice to quit; quitting has positive health benefits; hospital stay is an opportunity to quit; TEAM smoking cessation booklets contain helpful information." The statement, "I delivered cessation advice," was followed by space for the physician to sign.

SURVEYS AND CHART RECORDS

Before randomisation, patients were interviewed by research assistants for baseline information. Patients were surveyed again by telephone within 7-18 days of their discharge from the hospital. Phone surveys were administered by a team of trained telephone interviewers in the division of epidemiology at the University of Minnesota. Other information was collected from patient charts after hospital discharge.

\section{MEASURES}

The baseline survey was used to identify demographic and smoking related variables. The demographic variables used in this study were sex, age, ethnicity, educational level, and marital status. Smoking related variables included stage of change, addiction, health beliefs, and self efficacy. Stage of change is a measure of a smoker's readiness to quit smoking. ${ }^{22}$ Stages are classified as precontemplation (do not want to quit), contemplation (want to quit in next six months), preparation (want to quit in next 30 days, have made 24 hour quit attempt in past year), and action (have quit in past six months). Addiction was determined by the length of time to first morning cigarette, which has been found to be a good proxy of the more extensive Fagerstrom test for nicotine dependence. ${ }^{23}$ Health beliefs were determined by the question, "To what extent is smoking related to why you have been hospitalised? Would you say it is not at all related, somewhat related, or very much related?". Self efficacy is a measure of a smoker's confidence in being 
able to quit smoking, self rated on a scale from 0 to 10 .

The telephone interview was used to determine patients' quit attempts and their report of counselling received in the hospital. Patients were considered to have made a quit attempt if they reported they had not smoked in the last seven days or had tried to quit and stayed off cigarettes for at least 24 hours since leaving the hospital. To determine patient report of smoking cessation counselling, patients were asked whether physicians had done any of the following: "ask you if you smoked"; "ask you questions about your smoking, such as how long you have been smoking or how much you smoked"; "recommend you stop smoking/encourage you stay off cigarettes"; "tell you about the harmful effects of smoking"; "talk with you about how to quit or stay off cigarettes"; "give you written information about how to quit or stay off cigarettes"; or "suggest you get some help to stay off cigarettes after you were discharged from the hospital". A positive answer to any one or more of these seven questions was coded as patient reported counselling. We also created two alternate definitions of patient reported counselling: a five item definition excluding the first two questions, as asking about smoking was not specifically described on the physician chart label as "cessation advice"; and a single item definition, using only an affirmative answer to the question "recommend you stop smoking/encourage you to stay off cigarettes". The time to interview variable (from discharge to interview) was calculated in days.

The remaining information was taken from the patient chart. Length of hospital stay was calculated as hours from admission to discharge. Physician documentation of counselling was indicated by a physician's signature on the chart label; documentation that may have been present elsewhere in the chart was not included. Smoking related disease was determined by the discharge diagnosis, listed as an ICD-9 CM code (International Classification of Diseases, ninth revision, clinical morbidity). Any primary or secondary discharge diagnosis that has been identified as directly related to smoking was categorised as smoking related. ${ }^{24-26}$

ANALYSIS

Basic frequencies and correlations were calculated using SAS statistical software. ${ }^{27}$ Correlation was calculated using both Pearson's equation and the equation for $\kappa$, which takes the effects of chance into account.

Table 1 Patient reported and physician documented advice and correlation between reports

\begin{tabular}{|c|c|c|c|c|c|}
\hline & Total & Hospital A & Hospital B & Hospital C & Hospital D \\
\hline $\begin{array}{l}\text { Physician charted } \\
\text { advice }\end{array}$ & $\begin{array}{l}46.2 \% \\
\mathrm{n}=653\end{array}$ & $\begin{array}{l}37.5 \% \\
\mathrm{n}=104\end{array}$ & $\begin{array}{l}41.0 \% \\
\mathrm{n}=305\end{array}$ & $\begin{array}{l}45.8 \% \\
\mathrm{n}=142\end{array}$ & $\begin{array}{l}71.6 \% \\
\mathrm{n}=102\end{array}$ \\
\hline $\begin{array}{l}\text { Patient reported } \\
\text { advice }\end{array}$ & $\begin{array}{l}71.0 \% \\
\mathrm{n}=682\end{array}$ & $\begin{array}{l}71.9 \% \\
\mathrm{n}=114\end{array}$ & $\begin{array}{l}67.4 \% \\
\mathrm{n}=316\end{array}$ & $\begin{array}{l}80.7 \% \\
\mathrm{n}=150\end{array}$ & $\begin{array}{l}66.7 \% \\
\mathrm{n}=102\end{array}$ \\
\hline $\begin{array}{l}\text { Pearson } \\
\text { correlation }\end{array}$ & $\begin{array}{l}0.15 \\
p=0.0001\end{array}$ & $\begin{array}{l}0.19 \\
p=0.0575\end{array}$ & $\begin{array}{l}0.19 \\
p=0.0010\end{array}$ & $\begin{array}{l}0.10 \\
p=0.2360\end{array}$ & $\begin{array}{l}0.15 \\
p=0.1230\end{array}$ \\
\hline$\kappa$ & 0.13 & 0.14 & 0.16 & 0.08 & 0.15 \\
\hline
\end{tabular}

Modelling analysis of data was first done using the SAS/STAT MIXED procedure, followed by analysis with the SAS/STAT GLIMMIX macro, mixed model regression program. ${ }^{27}$ The adjusted probabilities reported here were obtained from the MIXED analyses, and the F tests and $\mathrm{p}$ values were obtained from the GLIMMIX analysis, which is more appropriate for analysis of dependent variables with dichotomous outcomes. The modelling for the outcome variables was first performed using only those independent variables obtained from the baseline interview (discharge diagnosis was considered a baseline variable, as a smoking related disease would likely be present upon admission to the hospital). The baseline variables found to be significant predictors were then put into a new model with variables occurring later in time (during or after hospitalisation). This was done to ensure that variables selected from sequential surveys were not predictors of each other. The final model was run using only those variables found to be significant in both analyses.

\section{Results}

SAMPLE

A total of 55115 patients were screened for this study, of which 13529 were smokers; 5547 were eligible to be in the study, 4370 were approached, and 2478 were enrolled. Of these, 2357 subjects completed the baseline survey; 790 were randomly assigned to the PA intervention, $682(86 \%)$ of whom completed the telephone interview. Of those who completed the telephone interview, 53.5\% were female, $78.5 \%$ were white, $14.9 \%$ were African American, 48.7\% had attended some college or vocational training, and $55.6 \%$ were married or in a marriage-like relationship. The mean patient age was 48 years. The median length of stay in the hospital was 73 hours; $35.6 \%$ of patients had a primary or secondary discharge diagnosis that was directly related to smoking and $38.4 \%$ thought that their hospitalisation was somewhat or very much related to smoking.

SMOKING CESSATION ADVICE REPORTED

Table 1 presents the patient reported and physician documented counselling, as well as the two calculations of correlation. Overall, $71.0 \%$ of patients reported smoking cessation counselling, while physicians documented counselling in the charts of $46.2 \%$ of patients. This pattern was found at all hospitals, with the exception of hospital D, where the rate of physicians charting counselling exceeded that of patients reporting it. The Pearson correlation between patient report and physician documentation was $0.15(\mathrm{p}=0.0001)$ over all hospitals, while $\kappa=0.13$. We also performed the analysis using both the five item and one item definitions of patient reported counselling. Using these more conservative definitions, $60.3 \%$ and $55.0 \%$ of patients reported counselling and the correlations between patient report and physician documentation were $0.20(p=0.0001)$ and $0.16(p=0.0001)$, respectively. Because these changes in 
definition did not greatly improve the correlation, subsequent analyses were conducted using the seven item version of patient reported counselling.

In regard to specific physician counselling behaviours, $59.8 \%$ of patients indicated that their physician asked if they smoked. For this question, patients were also given the option of answering that their provider already knew they smoked, but they were not asked to identify the provider; this made it impossible to do an analysis of physicians specifically. Patients also reported the following counselling behaviours: their physician recommended that they stop smoking, 55.0\%; asked questions about their smoking history, $41.9 \%$; and told them about the harmful effects of smoking, $33.9 \%$. Patients reported the following assistance with cessation: their physician talked about how to quit or stay off cigarettes, $15.2 \%$; recommended that they get help to stay off cigarettes after discharge, $14.1 \%$; and gave written information about how to quit or stay off cigarettes, $3.5 \%$.

PREDICTORS OF PATIENT REPORT OF COUNSELLING

Table 2 presents significant predictors of patient report of counselling. A separate analysis was also performed without "patient believes hospitalisation smoking related" included, to ensure that its possible high corre- lation with other variables, such as stage of change or self efficacy, did not mask their significance; the results did not change. Significant predictors of patient reported counselling were hospital, belief that hospitalisation was smoking related, smoking related disease, and corresponding documentation by the physician. Analyses were also conducted with the five item and one item definitions of patient reported counselling, with no difference in predictors identified.

PREDICTORS OF PHYSICIAN CHARTING COUNSELLING

Table 3 presents significant predictors of physician documentation of counselling. Four variables were found to be predictors: sex, with physicians charting more for females than males; hospital, with physicians at hospital D most likely to report counselling; and length of stay, with physician charting counselling as stays got longer. Smoking related disease was found to be a marginally significant predictor.

PREDICTORS OF A QUIT ATTEMPT AFTER HOSPITALISATION

Only physician and patient reports of counselling were analysed in the first model to determine if either was a significant predictor of a smoking cessation attempt alone (degrees of freedom 1, 650). Only patient report of counselling was found to be significant; $69.1 \%$

Table 2 Predictors of patient report of advice

\begin{tabular}{|c|c|c|c|c|c|c|}
\hline Variable & $n$ & $\begin{array}{l}\text { Proportion of } \\
\text { patients reporting } \\
\text { advice }\end{array}$ & Standard error & $\begin{array}{l}\text { Degrees of } \\
\text { freedom }\end{array}$ & $F$ & $p$ Value \\
\hline \multicolumn{7}{|l|}{ Hospital } \\
\hline A & 103 & 0.772 & 0.042 & 3,639 & 3.46 & 0.0162 \\
\hline $\mathrm{B}$ & 304 & 0.681 & 0.024 & & & \\
\hline $\mathrm{C}$ & 141 & 0.799 & 0.036 & & & \\
\hline $\mathrm{D}$ & 100 & 0.658 & 0.043 & & & \\
\hline \multicolumn{7}{|c|}{ Patient believes hospitalisation smoking related } \\
\hline Not at all & 361 & 0.618 & 0.023 & 3,639 & 10.64 & 0.0001 \\
\hline Somewhat & 134 & 0.844 & 0.037 & & & \\
\hline Very much & 115 & 0.821 & 0.042 & & & \\
\hline Don't know & 38 & 0.901 & 0.068 & & & \\
\hline \multicolumn{7}{|c|}{ Smoking related disease } \\
\hline Yes & 320 & 0.770 & 0.025 & 1,639 & 6.94 & 0.0086 \\
\hline No & 328 & 0.667 & 0.025 & & & \\
\hline \multicolumn{7}{|c|}{ Physician charts advice } \\
\hline Yes & 351 & 0.788 & 0.025 & 1,639 & 13.79 & 0.0002 \\
\hline No & 297 & 0.658 & 0.023 & & & \\
\hline
\end{tabular}

Table 3 Predictors of physician documentation of advice

\begin{tabular}{|c|c|c|c|c|c|c|}
\hline Variable & $n$ & $\begin{array}{l}\text { Proportion of } \\
\text { physicians } \\
\text { documenting advice }\end{array}$ & Standard error & $\begin{array}{l}\text { Degrees of } \\
\text { freedom }\end{array}$ & $F$ & $p$ Value \\
\hline \multicolumn{7}{|l|}{ Patient sex } \\
\hline Female & 346 & 0.498 & 0.026 & 1,641 & 5.61 & 0.0181 \\
\hline Male & 302 & 0.407 & 0.028 & & & \\
\hline \multicolumn{7}{|l|}{ Hospital } \\
\hline A & 103 & 0.382 & 0.048 & 3,641 & 10.24 & 0.0001 \\
\hline B & 304 & 0.401 & 0.028 & & & \\
\hline C & 141 & 0.452 & 0.041 & & & \\
\hline $\mathrm{D}$ & 100 & 0.715 & 0.048 & & & \\
\hline Length of hospital stay & 648 & $0.0141 /$ day $^{\star}$ & $0.005 /$ day & 1,641 & 4.69 & 0.0308 \\
\hline \multicolumn{7}{|l|}{ Smoking related disease } \\
\hline Yes & 320 & 0.492 & 0.027 & 1,641 & 3.45 & 0.0639 \\
\hline No & 328 & 0.421 & 0.027 & & & \\
\hline
\end{tabular}

*With each day in the hospital, the proportion of physicians documenting advice increased by this amount. 
(standard error $(\mathrm{SE})=0.022)$ of patients who reported counselling made a quit attempt, while only $57.1 \%(\mathrm{SE}=0.035)$ of patients who did not report counselling made a quit attempt $(\mathrm{F}=8.22, \mathrm{p}=0.0043)$. When physicians documented counselling, $67.6 \%$ $(\mathrm{SE}=0.027)$ of patients made a quit attempt, versus $64.0 \%(\mathrm{SE}=0.025)$ when physicians did not document counselling ( $F=0.94$, $\mathrm{p}=0.3330)$. Therefore, physician charted counselling was excluded from further models. In the final model, patient report of counselling was still found to be a significant predictor $(\mathrm{F}=7.09, \mathrm{p}=0.0080)$ of a smoking cessation attempt after adjusting for age group, stage of change, time to first morning cigarette, patient belief that hospitalisation is smoking related, hospital, and length of stay. Again, analyses were also done using the five item and one item definitions of patient reported counselling, with no difference in predictors identified.

\section{Discussion}

This study examined the rates of counselling documented by physicians and reported by patients, as well as the correlation between these two reports. It identified predictors of physician and patient reports of counselling, and the effect of reports of counselling on a smoking cessation attempt after discharge from the hospital.

SMOKING CESSATION COUNSELLING REPORTED The frequencies of counselling reported indicate that patients report counselling at a higher rate than that indicated by physician documentation in the chart. This was not surprising, given the results of previous studies. ${ }^{14-21}$ What is surprising is the extremely low correlation between the two reports. Although the relationship between patient report and physician documentation was significant, the correlation was only $13-15 \%$. It is possible that this discrepancy is at least in part an artefact of the way counselling was defined for the two groups in this study. Patients were asked seven specific questions about the content of their discussions with physicians; the term "counselling" was not left to their interpretation. Physicians, on the other hand, were directed to have a discussion with their patient that "included" four items; they were not asked to specifically report what had been done. The statement, "I delivered cessation advice", may have been interpreted in multiple ways, making comparison between the two reports potentially problematic.

It is also important to recognise that the poor correlation may have been the result of faulty patient recall and over report, or failure of the physician to document all cases in which counselling was performed. This may simply be the nature of patient report and physician documentation. However, the length of time from discharge to the phone interview may have biased patient report. An exit interview might be more appropriate to ensure accurate reporting. However, length of time to phone interview was not a significant predictor of patient report. Physician documentation may have been affected by the use of chart labels. They were intended to make documentation easier, yet counselling may have been recorded elsewhere, precluding inclusion in our analysis. While our results may reflect the poor feasibility of the use of chart labels in the hospital, it is also possible that results would be worse without a "user friendly" system in place.

Regardless of the cause, the poor correlation between patient report and physician documentation has implications in both the clinical and the research setting. For counselling to be maximally effective clinically, it is important that both the patient and the physician perceive that counselling has been done. In the context of research, it is important to realise that the source of a report of counselling must be considered in analysis; patient and physician reports do not seem to be interchangeable variables.

Other studies have offered solutions to resolve the disagreement between patient report and physician documentation. Brown and Adams simply calculate the average incidence of advice reported. ${ }^{17}$ Cummings and colleagues simply disregard physician documentation, as it is the patient who must ultimately make a health behaviour change. ${ }^{16}$ Wilson and McDonald conclude that patient surveys are most reasonable for large scale research studies, owing to high sensitivity rates and despite high false positive rates. ${ }^{15}$ Ultimately, none of these solutions seems satisfactory, as they do not take the relative accuracy of both reports into account.

We suggest that further research is warranted in order to determine the true rate of smoking cessation counselling in the inpatient setting, as well as to verify trends in patient report and physician documentation. The inpatient setting poses some particular challenges to a research study such as this, as patients have encounters with many physicians multiple times per day. It is unlikely that every encounter could be audio- or videotaped. It would be more reasonable to record interactions during a specific time, such as during morning rounds. Given the possible complications in our study as a result of different definitions of counselling, we also recommend that patients and physicians be asked identical questions about the content of the counselling.

Finally, we find the high rate of physician documentation at hospital D worth comment; it contradicts the trends found at all other hospitals in the study, as well as those in the literature to date. This hospital was quite zealous in promoting this research project, which may have resulted in higher physician documentation rates.

TYPES OF COUNSELLING REPORTED BY PATIENTS According to patient report, physicians are doing a moderate job of asking their patients about smoking, informing them of the harmful effects of smoking, and advising them to quit. However, they are doing a very poor job of providing information about how to quit. The low rate of patients reporting receiving written 
information may have been caused by physicians' awareness that the TEAM booklet had already been given. Nevertheless, only $15.2 \%$ of patients reported talking with their physician about how to quit or stay off cigarettes, and only $14.1 \%$ reported that their physician recommended that they get help to stay off cigarettes after discharge. As a hospital stay presents a "teachable moment", physicians should be taking advantage of this opportunity to educate their patients on how to quit smoking.

PREDICTORS OF REPORTS OF COUNSELLING

Patients were more likely to report counselling when they believed that their hospitalisation was related to their smoking, they had a smoking related disease, or their physician had charted counselling. Patients in hospitals A and C were also more likely to report counselling. Health beliefs and smoking related disease could act as mediators of patient self report in several ways. For instance, these patients may have been listening for counselling more closely, making them more likely to note and report it. Conversely, they may have been so expectant of receiving counselling that they incorrectly assumed it was given. In either case, patients with smoking related illnesses and hospitalisations may be more open to discussing smoking cessation; physicians should be conducting interventions with this group.

Physicians were more likely to chart counselling when the patient was female, they were at hospital D, the length of stay was greater, and the discharge diagnosis was smoking related. As indicated previously, sex has been shown to predict patient report of advice $^{81213}$; this has been attributed to factors such as more frequent clinic visits by women and the known increased risk of thromboembolism with concurrent use of oral contraceptives. With a longer hospital stay, the patient was likely more ill; this might prompt physicians to view discussion and subsequent documentation of smoking as a more important aspect of treatment. Also, the longer the stay, the more opportunities physicians had to see the chart label and respond to it. In the context of a smoking related disease, physicians may have been more apt to view counselling as a vital part of patient care, making it more likely they would both give and document counselling in the chart.

It is also important to note factors which were not identified as predictors of physician documentation. For instance, patients in the preparation stage of change are ready to quit smoking, but stage of change was not a predictor of documentation. This is an unfortunate missed opportunity, as patients in preparation may successfully quit smoking with clear, consistent instruction on how to quit. In the hospital, documentation is important in ensuring that this takes place. It is often the only way that many physicians are made aware of counselling, allowing them to reinforce each others' messages. Furthermore, in order for primary care physicians to continue care effectively after discharge, it is important that they know that counselling has taken place.

\section{PREDICTORS OF OUIT ATTEMPT}

We examined reports of counselling as predictors of cessation and found that only patient reported counselling predicted a quit attempt. This seems logical, as it is ultimately the patient who must be motivated to make the health behaviour change. This has important implications; if the patient report is the only one that predicts a cessation attempt, physicians should be making efforts to ensure that patients will recall their counselling.

While we feel that our study has demonstrated the importance of patient report of counselling in smoking cessation, it is also important to note some limitations not already discussed above. At this point, we have not biochemically confirmed reported quit attempts. Furthermore, we do not yet have long term abstinence results, so no statements can be made about the impact of reports of counselling on long term cessation.

\section{CONCLUSION}

In the hospital setting, patients report smoking cessation counselling at a higher rate than physicians document it. Furthermore, reports of counselling are very poorly correlated. A patient report of counselling is predicted by specific study hospital, patient belief that the hospitalisation is smoking related, a smoking related disease, and documentation of counselling by the physician. Physician reports of counselling are more likely when patients are female, admitted to a specific hospital, have a longer hospital stay, and have a smoking related disease. The only report of counselling that was a predictor of a quit attempt was that of the patient. Given this finding, physicians should be taking steps to ensure that their patients understand and recall the counselling that they provide.

We thank Rose Hilk for her assistance and guidance in data management and analysis. This research was supported by grant HL54132 from the National Institutes of Health.

1 Emmons KM, Goldstein MG. Smokers who are hospitalized: a window of opportunity for cessation interventions. ized: a window of opport

2 Neighbor WE, Stoop DH, Ellsworth A. Smoking cessation counseling among hospitalized smokers. Am f Prev Med 1994;10:140-4

3 Houston Miller N, Smith PM, DeBusk RF, et al. Smoking cessation in hospitalized patients: results of a randomized trial. Arch Intern Med 1997;157:409-15.

4 Rigotti NA, Arnsten JH, McKool KM, et al. Efficacy of a smoking cessation program for hospital patients. Arch Intern Med 1997;157:2653-60.

5 Robinson MD, Laurent SL, Little JM. Including smoking status as a vital sign: it works. F Fam Pract 1995:40;556-61.

6 Dickey LL, Gemson DH, Carney P. Office system interventions supporting primary care-based health behavior change counseling. Am f Prev Med 1999;17:299308

7 Ockene JK, Hosmer DW, Williams JW, et al. The relationship of patient characteristics to physician delivery of advice to stop smoking. F Gen Intern Med 1987;2:337-40.

8 Frank E, Winkleby MA, Altman DG, et al. Predictors of physicians' smoking cessation advice. FAMA 1991; 266:3139-44.

9 Anda RF, Remington PL, Sienko DG, Davis RM. Are physicians advising smokers to quit? The patient's perspective. fAMA 1987;257:1916-19.

10 Gilpin EA, Pierce JP, Johnson M, Bal D. Physician advice to quit smoking: results from the 1990 California Tobacco Survey. F Gen Intern Med 1993;8:549-53. 
11 Goldstein MG, Niaura R, Willey-Lessne C, et al. Physicians counseling smokers: a population-based survey of patients perceptions of health care provider-delivered smoking ces-

sation interventions. Arch Intern Med 1997;157:1313-19.
Friedman C, Brownson RC, Peterson DE, Wilkerson JC. Physician advice to reduce chronic disease risk factors. $\mathrm{Am}$ F Prev Med 1994;10:367-71

13 Gilpin E, Pierce J, Goodman J, et al. Trends in physicians' giving advice to stop smoking, United States, 1974-87. Tobacco Control 1992;1:31-6.

14 Ward J, Sanson-Fisher R. Accuracy of patient recall of opportunistic smoking cessation advice in general practice. Tobacco Control 1996;5:110-13.

15 Wilson A, McDonald P. Comparison of patient questionnaire, medical record, and audio tape in assessment of health promotion in general practice consultations. $B M \mathcal{F}$ 1994;309:1483-5.

16 Cummings KM, Giovino G, Sciandra R, et al. Physician advice to quit smoking: who gets it and who doesn't. $A m \mathcal{F}$ advice to quit smoking: who

17 Brown JB, Adams ME. Patients as reliable reporters of medical care process: recall of ambulatory encounter 2;30:400-11.

18 Rethans JJ, Martin E, Metsemakers J. To what extent do clinical notes by general practitioners reflect actual medical performance? A study using simulated patients. $\mathrm{Br}$ f Gen Pract 1994;44:153-6.

19 Pbert L, Adams A, Quirk M, et al. The patient exit interview as an assessment of physician-delivered smoking intervention: a validation study. Health Psychol 1999;18:183-8.
20 Stange KC, Zyzanski SJ, Smith TF, et al. How valid are medical records and patient questionnaires for physician profiling and health services research? A comparison with direct observation of patient visits. Medical Care 1998; 36:851-67

21 Gerbert B, Hargreaves WA. Measuring physician behavior. Medical Care 1986;24:838-47.

22 Prochaska JO, DiClemente CC. Stages and processes of self-change of smoking: toward an integrative model of change. F Consult Clin Psychol 1983;51:390-5.

23 Heatherton TF, Kozlowski LT, Frecker RC, Fagerstrom $\mathrm{KO}$. The Fagerstrom test for nicotine dependence: a revision of the Fagerstrom tolerance questionnaire. $\mathrm{Br} F$ Addiction 1991;86:1119-27.

24 Centers for Disease Control and Prevention. Cigarette smoking-attributable mortality and years of potential life lost - United States, 1990. MMWR Morb Mortal Wkly Rep 1993;42:645-9.

25 Shopland DR. Tobacco use and its contribution to early cancer mortality with a special emphasis on cigarette smoking. Environ Health Perspect 1995;103:131-42.

26 US Department of Health and Human Services. Reducing the health consequences of smoking: 25 years of progress. A report of the Surgeon General, 1989. Rockville, Maryland: Public Health Service, Centers for Disease Control, Office on Smoking and Health, 1989. (DHHS Publication No (CDC) 89-8411.

27 SAS Institute. SAS/STAT software: changes and enhancements, through release 6.12. Cary, North Carolina: SAS Institute, 1997.

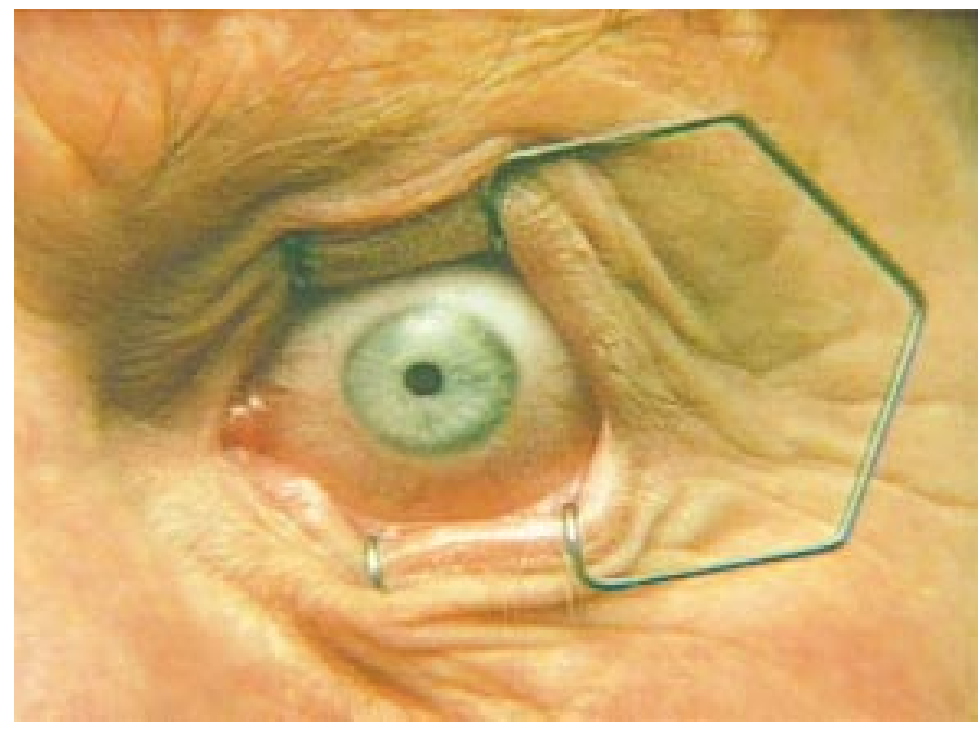

Stop it ... or you'll go blind: an image from the latest Australian national Quit campaign, warning smokers about age related macular degeneration (blindness) caused by smoking. The theme was adopted following an editorial in the Medical fournal of Australia recommending that smokers be advised of this little known, but well established risk (see Mitchell P, Chapman S, Smith W. "Smoking is a major cause of blindness": a new cigarette pack warning? Med f Aust 1999;171:173-4). 